\title{
Zum 60. Geburstag von Heinrich Bueß
}

Professor Dr. Heinrich Bueß ist am 17. Mai 1911 in St. Chrischona bei Bettingen geboren. Er hat den größten Teil seines arbeitsreichen Lebens in Basel verbracht. Dort ist er aufs humanistische Gymnasium gegangen, dort hat er studiert, dort war er 1937-38 Assistent auf der Pathologischen Anatomie und 1938-42 im Frauenspital, wo er den F M H für Gynäkologie und Geburtshilfe erwarb. In diese Zeit fallen auch 600 Tage Aktivdienst. Am 8. Oktober 1942 heiratete er. Er ist der Vater von vier Söhnen und einer Tochter. Von 1942 bis 1946 studierte er dann noch Geschichte und habilitierte sich 1946 für das Fach der Medizingeschichte. Seit 1946 ist er auch Fabrikarzt der сів A. 1949 war er Gastprofessor an der freien Universität Berlin. 1951/52 weilte er in den USA, wo der Endesunterzeichnete das erstemal das Privileg hatte, mit Heinrich Bueß zusammenzuarbeiten. 1950 erhielt Bueß einen Lehrauftrag für Medizingeschichte in Basel. Bis 1947 war der Unterricht vor allen Dingen von seinem uns allen teuren Lehrer Johann Karcher versehen worden. 1956 wurde Bueß außerordentlicher Professor. 1964 leitete er den Internationalen Kongreß für Medizingeschichte in Basel. Seither ist er auch Direktor der medizinhistorischen Bibliothek der Universität. Er ist Mitglied und Ehrenmitglied zahlreicher wissenschaftlicher Gesellschaften, vor allen Dingen auch Vizepräsident unserer Schweizerischen Gesellschaft. Er ist Gründungsmitglied der Schweizerischen Gesellschaft der Fabrik- und Betriebsärzte, deren Präsident er bis 1970 war.

Es ist selbstverständlich, daß bei nicht weniger als 220 vorliegenden wissenschaftlichen Arbeiten unseres Jubilars hier keine Detailanalysen vorgenommen werden können, sondern nur die Tendenzen und Höhepunkte von Heinrich Bueß wissenschaftlicher Arbeit aufgezeigt werden können. Auch fällt nur das medizinhistorische, nicht das berufsärztliche Werk des Jubilars in meinen Kompetenzbereich.

Bueß begann seine historische Tätigkeit 1938 mit einer Bibliographie zur Pathologischen Anatomie. Während seine psychiatriegeschichtliche Doktorarbeit sein einziger Beitrag auf diesem Gebiet blieb, ist er auch späterhin wiederholt zur Geschichte der Pathologischen Anatomie zurückgekehrt, insbesondere zur Pathologischen Anatomie des 17. Jahrhunderts. Den Höhepunkt dieser Bemühungen stellt wohl die mit Joos und Portmann 1961 veröffentlichte Studie über die pathologisch-anatomischen Arbeiten bedeutender Schweizer Ärzte 1670-1720 dar.

Dies ist durchaus nicht der einzige Aspekt der Medizin des 17.Jahrhunderts, den Bueß erfolgreich bearbeitet hat. Im Gegenteil, er kann wohl als einer der besten Kenner der Medizingeschichte dieser Periode, die er gern als Barockmedizin charakterisiert, bezeichnet werden.

Auch seine Habilitationsschrift Die historischen Grundlagen der intravenösen Injektion (1946), eine der wertvollsten medizinhistorischen Publikationen der 
letzten Jahrzehnte, beschäftigt sich ja mit einem wichtigen Problem dieses Jahrhunderts. Gleichzeitig mit der Injektion hat Bueß auch begonnen, die Geschichte von Thrombose und Embolie zu behandeln, zu der er bis 1967 immer wieder zurückgekehrt ist. Nahe verwandt diesen Forschungen sind auch jene über Geschichte der Hämodynamik und Pathologischen Physiologie, in denen sich Bueß vor allen Dingen dem Werk Albrecht Hallers zugewendet hat (siehe z. B. Gesnerus 1954, 1957, 1958).

Auch bei seiner Beschäftigung mit der Medizingeschichte Basels, welche eine andere große Gruppe im Werk von Bueß und seinen Schülern darstellt, ist Bueß immer wieder zu Barockproblemen in Basels Medizingeschichte zurückgekehrt. Erwähnt werden sollten hier auch seine Beiträge zur Geschichte des Bürgerspitals und der Medizinischen Gesellschaft.

Da Bueß als Geburtshelfer und Gynäkologe ausgebildet wurde, ist es nicht verwunderlich, daß er seit 1948 zahlreiche Beiträge zur Geschichte dieser Disziplinen geleistet hat, vor allem die 1970 erschienene Arbeit Geburtshilfe und Gynäkologie in Basel 1868-1942. Sogar schon seit 1942 hatte er sich erfolgreich Problemen der Geschichte der: Entwicklungs-Physiologie zugewendet.

Ebenso organisch ergab es sich, daß ein aktiver Arbeitsmediziner wie Bueß die Geschichte seines Faches bearbeitete. Bueß hat auch seit 1942 Beiträge zur Geschichte der Militärmedizin geleistet.

Als kleinere Arbeitsgebiete, auf denen Bueß Wertvolles geleistet hat, seien die Pharmakotherapie (z. B. Gesnerus 1953, 1962), die Geschichte der Transfusion und vor allem auch seine Arbeiten zur Historiographie erwähnt. Schließlich bleibt die Schrift Schweizer Ärzte als Forscher, Entdecker und Frfinder, Basel 1945, die beste Zusammenfassung, welche wir zur Geschichte der Schweizer Medizin besitzen.

Das wissenschaftliche Werk von Bueß ist quantitativ und qualitativ gleich eindrucksvoll. Sein Euvre übertrifft das meiste, was im deutschsprachigen Gebiet in den letzten Jahrzehnten geleistet worden ist.

$\mathrm{Zu}$ diesen persönlichen Arbeiten von $\mathrm{Bue} ß$ treten jetzt noch die 31 Arbeiten, die in seinem Institut abgefaßt wurden, und die 46 Dissertationen, welche unter seiner Anregung und Betreuung entstanden. Unter letzteren befinden sich weit überdurchschnittlich wertvolle Arbeiten, wie die von Bornhauser, Möschlin, Mani, Thüer, Reiner, Eichenberger, Haller, Wobmann und Häfliger. Es ist kein Zufall, daß die letzten drei Arbeiten mit dem Henry-E.-Sigerist-Preis der Schweizerischen Gesellschaft für Geschịchte der Medizin und Naturwissenschaften ausgezeichnet wurden.

Heinrich Bueß hat sich wissenschaftlich und organisatorisch während mehr als drei Jahrzehnten um die Schweizer Medizingeschichte wohl verdient gemacht. Dafür wollen wir ihm anläßlich seines 60 . Geburtstags herzlich danken und ihm das Beste für die Zukunft wünschen.

Erwin H. Ackerknecht 